\title{
Perda auditiva e hipertensão: achados em um grupo de idosos
}

\section{Hearing loss and hypertension: findings in an older by group}

\author{
Giovana S. Baraldi' ${ }^{1}$, Laís C. Almeida², \\ Alda Cristina L. C. Borges ${ }^{3}$
}

Palavras-chave: pressão alta, audição, idoso.

Key words: high pressure, hearing, aged.

\section{Resumo / Summary}

om o avanço da idade cresce o número de doenças crônicas sendo a hipertensão arterial sistêmica (HAS) e a perda auditiva de grande prevalência na população idosa. Objetivo: Comparar e analisar os resultados de anamnese e audiometria tonal limiar de pacientes idosos hipertensos e não-hipertensos. Forma de estudo: Estudo de coorte contemporânea transversal. Material e Método: Este estudo foi realizado na UNIFESP no período de março a novembro de 2003. Foram avaliados através de anamnese e audiometria tonal limiar 70 idosos, com idade entre 60 e 92 anos, sendo 15 do sexo masculino e 54 do sexo feminino. Resultados: Q uanto aos achados audiológicos dos grupos estudados podese observar diferença com relação à configuração audiométrica do tipo rampa e a queixa de "zumbido", ambas mais freqüentes no grupo dos idosos não-hipertensos. Conclusão: Os idosos hipertensos não apresentaram maior déficit auditivo, sendo o grau de perda de audição semelhante nos dois grupos. A configuração audiométrica predominante no grupo de idosos hipertensos foi do tipo plana, enquanto que nos não-hipertensos foi do tipo rampa. Com relação às queixas audiológicas, o grupo de não-hipertensos apresentou maior ocorrência da queixa "zumbido".

\begin{abstract}
$A$ the age advances, the number of chronic diseases also grows, the systemic arterial hypertension (SAH) and hearing loss having a significant prevalence in aged people. Objective To compare and analyze anamnesis and threshold tonal audiometry results in both hypertensive and nonhypertensive patients. Study design: Transversal contemporany cohort study. Material and Method: This study has been conducted at UNIFESP in the period from March to November 2003. Seventy people in the age group from 60 to 92 - 15 male and 54 female - were evaluated through anamnesis and threshold tonal audiometry. Results As to audiology findings of groups studied, a difference can be noted regarding "ramp-typed" audiometric configuration and buzz claim, both occurring most frequently in nonhypertensive aged people. Conclusion: Hypertensive aged people did not present a higher hearing deficit, the degree of hearing loss being similar in both groups. Prevailing audiometric configuration in hypertensive group was that of "full-type", while the non-hypertensive group presented a "ramp-typed" configuration. As to audiologic claims, the non-hypertensive group presented an increased occurrence of "buzz" claim.
\end{abstract}

\footnotetext{
${ }^{1}$ Fonoaudióloga especialista em Gerontologia pela Universidade Federal de São Paulo - Escola Paulista de Medicina/ Mestranda do Departamento de Fonodiaulogia da Universidade Federal de São Paulo - Ecola Paulista de Medicina.

${ }^{2}$ Fonoaudióloga especialista em Distúrbios da Comunicação pela Universidade Federal de São Paulo - Escola Paulista de Medicina/ Mestranda do Departamento de Fonodiaulogia da Universidade Federal de São Paulo - Ecola Paulista de Medicina. ${ }^{3}$ Professor adjunto INIFESP/EPM.

Endereço para correspondência: Giovana S. Baraldi - Praça Alberto, 87 04672-130 São Paulo SP

Tel (0xx11) 9773-3488 - E-mail: giovanafono@bol.com.br

Artigo recebido em 18 de maio de 2004. Artigo aceito em 19 de julho de 2004.
} 


\section{INTRODUÇÃO}

0 envelhecimento populacional vem fazendo parte do cenário mundial em função da progressiva elevação da esperança média de vida, relacionada à diminuição das taxas de mortalidade e natalidade. No Brasil, de acordo com o último Censo (2000), a população idosa correspondia a 5,85\% da população, sendo o crescimento de $1,02 \%$ em relação ao Censo anterior de 1991. 0 índice de envelhecimento também aumentou de 13,90\% em 1991 para 19,77\% em 2000.

Com o aumento da idade aumentam o número de doenças crônicas, sendo a hipertensão arterial sistêmica (HAS), um dos mais importantes fatores de risco cardiovascular ${ }^{1}$. A prevalência da HA em idosos, segundo dados americanos, indica que desde 1900 a percentagem de pessoas hipertensas acima de 65 anos triplicou $(4,1 \%$ para $13,0 \%$ ), e o número aumentou 10 vezes (3,1 para 31,2 milhões) ${ }^{2}$. A Hipertensão Arterial é defendida como uma PAS (pressão arterial sistólica) de $140 \mathrm{mmHg}$ ou maior e PAD (pressão arterial diastólica) de $90 \mathrm{mmHg}$, conforme determina o VI Joint National Committee ${ }^{3}$.

Ainda tratando-se de doenças crônicas, sabe-se que, em geral, a idade avançada está associada a um comprometimento maior dos sentidos, sendo a audição um dos sentidos essenciais para a comunicação. Sabe-se que a perda auditiva tem um efeito adverso no estado funcional, na qualidade de vida, na função cognitiva e no bem-estar emocional, comportamental e social do indivíduo idoso. Em alguns estudos, segundo a variável sexo e idade, utilizando indivíduos idosos há prevalência de $63 \%$ de deficiência auditiva.

A perda auditiva decorrente do envelhecimento é conhecida como presbiacusia e está associada a características audiológicas específicas, sendo a perda de audição neurossensorial, de configuração descendente, bilateral e simétrica. Segundo o Commitee on Hearing, Bioacoustics and Biomechanics, a presbiacusia foi definida como a soma de perdas auditivas que resulta de muitas variedades de degeneração fisiológica incluindo prejuízos causados pela exposição ao ruído, a agentes ototóxicos e prejuízos causados por desordens e tratamentos médicos, e tem como características funcionais uma mudança descendente gradual na sensibilidade auditiva para todas as freqüências, acompanhada por um decréscimo na discriminação da fala e um declínio complexo da função auditiva central que se manifesta através do aumento da dificuldade nas habilidades como fusão auditiva, atenção auditiva, julgamento auditivo, comportamentos variados e uma redução na velocidade de fechamento e síntese auditivos. Vários são os fatores envolvidos na sua etiologia como exposição a ruídos, dieta, hipertensão, fatores metabólicos e hereditários.

A relação PAS x PAD para eventos cardiovasculares é igualmente mais pronunciada em pessoas com idade maior que 65 anos, quando comparados a indivíduos com idade entre 35 a 64 anos $^{1}$. Por sua vez, a presbiacusia é definida em senso estrito como a perda auditiva avaliada em um indivíduo na quinta década de vida ou mais decorrente exclusivamente de mudanças relacionadas à idade possivelmente de base genética.

Portanto, pensando nas mudanças da atividade coclear que ocorrem em função de doenças metabólicas, e na prevalência da pressão alta e da perda auditiva em indivíduos idosos, acredita-se que a hipertensão pode ocasionar danos ao sistema auditivo. Devido a isto, a fim de prevenir e detectar os déficits auditivos advindos do envelhecimento, o objetivo deste estudo foi comparar e analisar os resultados de anamnese e audiometria tonal limiar de pacientes idosos hipertensos e não-hipertensos.

\section{MATERIAL E MÉTODO}

De acordo com as normas preconizadas para experiências utilizando seres humanos, este estudo foi analisado e aprovado pelo Comitê de Ética em Pesquisa da Universidade Federal de São Paulo conforme resolução CEP n- 0669/03 do Conselho Nacional de Saúde.

Com relação à amostra utilizada, participaram deste estudo idosos encaminhados pelo Instituto de Geriatria e Gerontologia da UNIFESP para realização de avaliação audiológica no período de março a novembro de 2003. Foram avaliados 70 idosos, com idade entre 60 e 92 anos, sendo 15 do sexo masculino e 54 do sexo feminino. 0 diagnóstico da hipertensão foi considerado segundo critério médico e utilizou-se apenas pacientes hipertensos controlados.

Os indivíduos foram submetidos inicialmente a uma anamnese, composta por questões relacionadas a hábitos nocivos ao sistema auditivo, acuidade auditiva e funcionamento deste sistema no meio social, como: dados referentes à dificuldade para compreender a fala em ambiente ruidoso e ao telefone; necessidade em aumentar o volume da TV; privação social em função do déficit auditivo; história otológica; presença de zumbido e tontura.

Após anamnese os idosos foram submetidos à avaliação audiológica básica, realizada em cabina acústica, incluindo os seguintes procedimentos:

- Audiometria Tonal Limiar: realizada em audiômetro Modelo AC33, de marca Interacoustics, calibrado segundo a norma ANSI 69.

- Audiometria Vocal: Limiar de reconhecimento de fala (SRT) e Índice percentual de Reconhecimento de fala (IPRF).

- Medidas de Imitância Acústica: Timpanometria e Pesquisa dos Reflexos Acústicos Contralaterais, realizada em Imitanciômetro Modelo AZ7, de marca Interacoustis.

- As audiometrias foram classificadas quanto ao grau como: leve, moderada, moderadamente severa, severa e profunda, segundo o critério de Lloyde e Kaplan 1978. 
- As configurações audiométricas foram classificadas como: plana, gradual, abrupta e rampa de acordo com critérios, da categoria principal, estabelecidos por Lloyde e Kaplan 1978, para classificação de curvas descendentes.

Para interpretação dos resultados obtidos foram utilizados os testes ANOVA, Teste de Igualdade de Duas Proporções, Teste de Qui-quadrado e também uma analise descritiva composta pelo Intervalo de Confiança.

\section{RESULTADOS}

De acordo com o objetivo deste estudo, os dados obtidos dos dois grupos estudados - idosos hipertensos e idosos não-hipertensos - foram comparados segundo: grau e configuração da perda auditiva e dados de anamnese. 0 nível de significância utilizado para as comparações estatísticas foi de 0,05 (5\%).

Inicialmente serão apresentados os resultados com uma análise descritiva completa para os graus de perda auditiva, tanto quem tem e não pressão alta. Vale lembrar que construiu-se os intervalos (limite inferior e superior) com 95\% de confiança estatística (gráfico 1 e 2).

Averiguamos que em nenhuma das orelhas existe diferença proporcional entre os grupos que seja estatisticamente significante em nenhum dos níveis de grau.

No gráfico 3 e 4 serão apresentados os resultados para os tipos de configuração audiométrica.

Existiu diferença proporcionalmente significativa entre os grupos para o nível de curva "rampa". Essa diferença aparece tanto na orelha direita quanto na esquerda. Verificando que o grupo dos que não tem pressão alta é de maior recorrência estatística deste tipo de curva.

No gráfico 5 serão apresentados os dados de anamnese que apresentaram diferença relevante com relação aos grupos estudados.

Entre Zumbido e HAS, verificamos que existe uma dependência e/ou associação estatística, sendo os nãohipertensos mais propensos a apresentar este tipo de quei$x a, p$-valor $=0,009 *$

\section{DISCUSSÃO}

No Brasil a hipertensão arterial é um dos mais importantes fatores de risco cardiovascular, acometendo cerca de $20 \%$ da população idosa acima de 65 anos de idade, na cidade de São Paulo, estudos epidemiológicos indicam uma prevalência ainda maior sendo de $62 \%$ entre os indivíduos idosos ${ }^{1}$. Por sua vez, a perda auditiva acomete cerca de $24 \%$ da população entre 65 e 74 anos de idade e $39 \%$ na população acima de 75 anos ${ }^{11}$.

A relação entre a hipertensão e perda de audição é vastamente estudada na literatura, através de dados clínicos e histopatológicos, sendo os achados controversos.

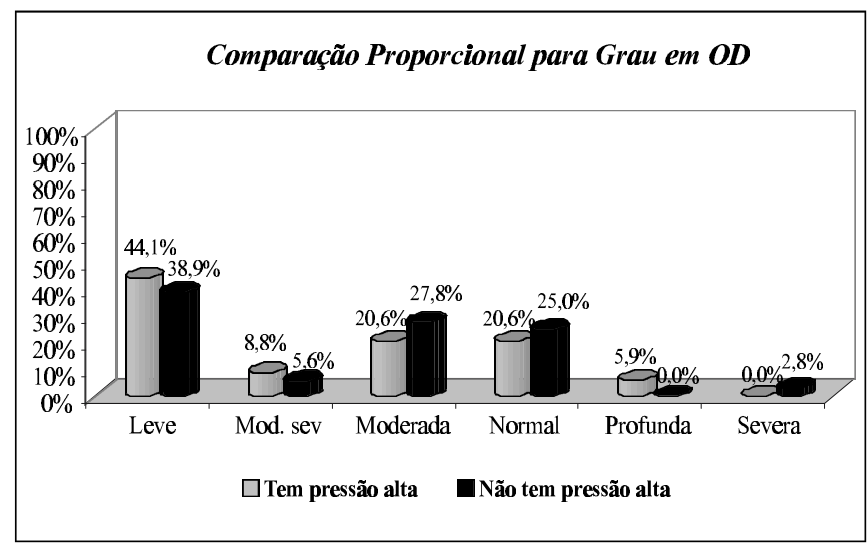

Figura 1. Distribuição dos graus de perda auditiva nos grupos estudados para orelha direita (OD).

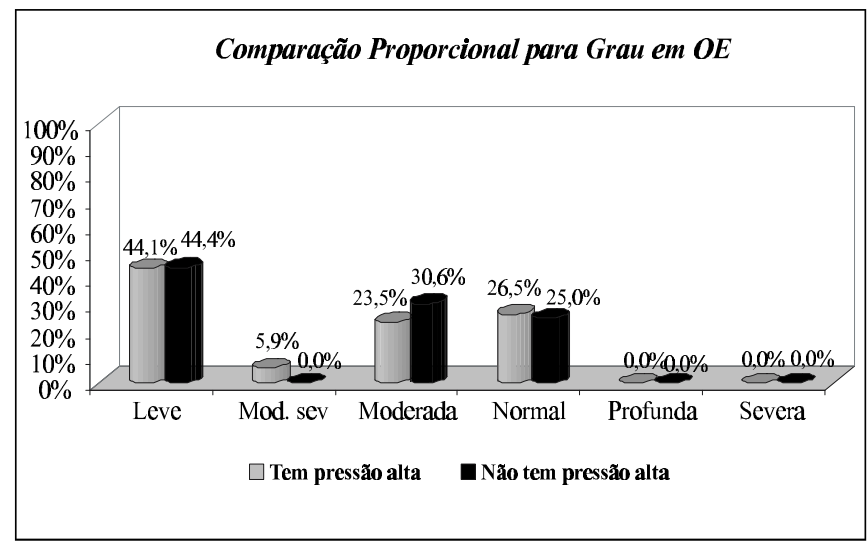

Figura 2. Distribuição dos graus de perda auditiva nos grupos estudados para orelha esquerda $(O E)$.

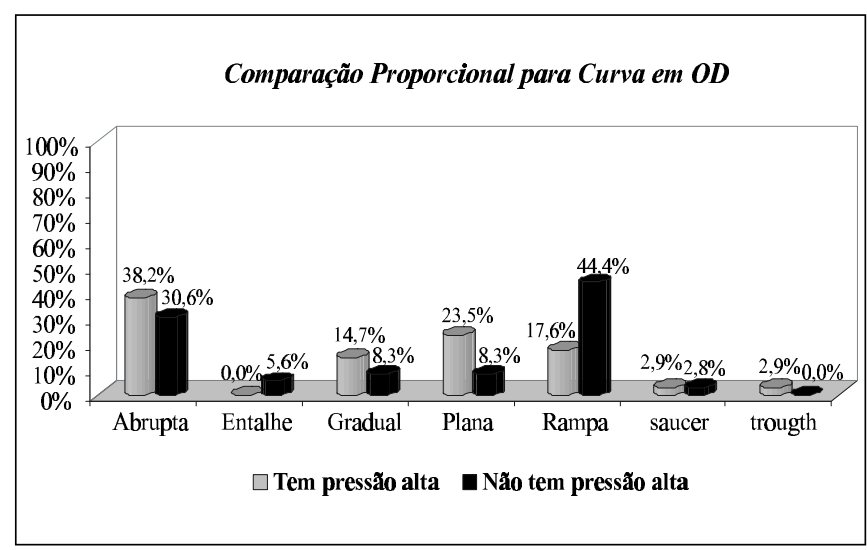

Figura 3. Distribuição dos tipos de configuração audiométrica nos grupos estudados na orelha direita. 


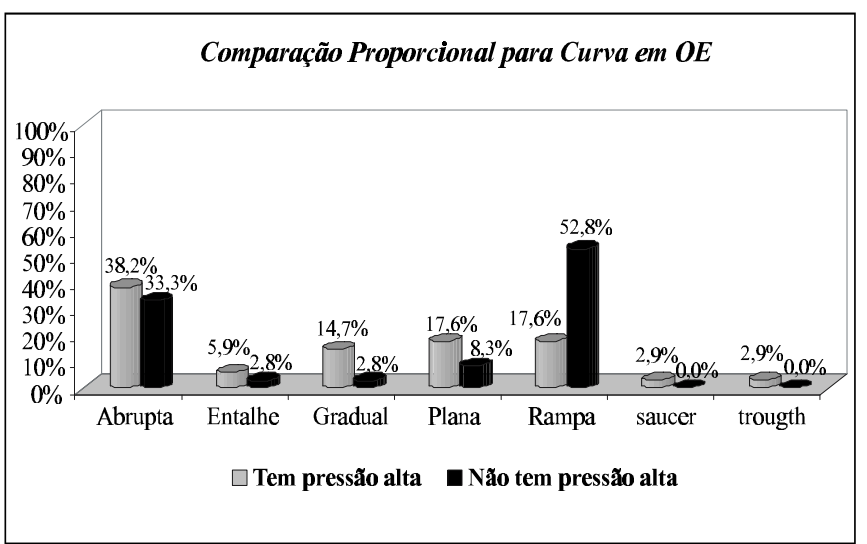

Figura 4. Distribuição dos tipos de configuração audiométrica nos grupos estudados na orelha esquerda.

Alguns estudos justificam que a surdez sensorioneural ocorreria em função de uma insuficiência microcirculatório decorrente de uma oclusão vascular por embolia, hemorragia ou vasoespasmo e que estes seriam produto de uma síndrome de hiperviscosidade ou microangiopatia por diabetes ou hipertensão, sendo postulado que a hipertensão poderia através desses fatores histopatológicos provocar perda de audição sensorioneural ${ }^{12}$. Já outros discutem que a HAS isolada não geraria perda de audição superior ao observado na população normotensa ${ }^{13}$ ao passo que a hipertensão associada a outros fatores como: tensão, exposição a ruído, predisposição genética poderia possivelmente ocasionar perda significativa na audição ${ }^{1}$.

Acreditando na estreita relação entre a hipertensão e a perda de audição, este estudo buscou pesquisar os achados audiológicos entre grupos de idosos hipertensos e nãohipertensos, sendo encontrado como primeiro dado que, com relação ao grau da perda auditiva não ocorreram diferenças entre os dois grupos estudados. Ao contrário de relatos encontrados na literatura, o grupo de idosos hipertensos não apresentou maior grau de perda de audição que dos idosos não-hipertensos ap resentando graus de perda auditiva semelhantes.

Já com relação aos tipos de configuração audiométrica estudados, existiu diferença proporcionalmente significativa entre os dois grupos, idosos hipertensos $\mathrm{x}$ idosos normotensos, para a curva do tipo "rampa". Essa diferença aparece tanto na orelha direita quanto na esquerda, verificando que a configuração do tipo rampa foi de maior recorrência estatística nos idosos normotensos. Ainda com relação à configuração audiométrica, observou-se que a configuração do tipo plana foi mais freqüente nos idosos hipertensos em comparação com os não-hipertensos, fato que é discutido na literatura, uma vez que evidências histológicas e achados audiológicos combinados podem delinear tipos específicos de presbiacusia sendo uma delas a

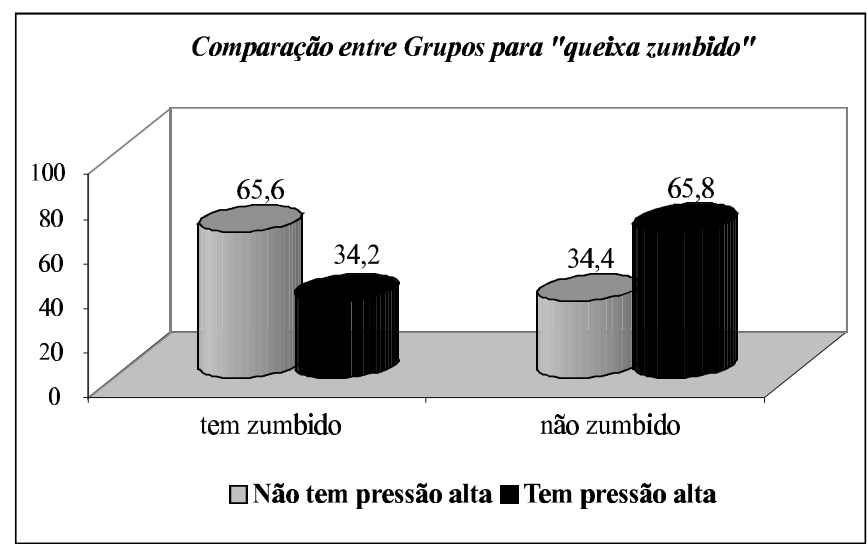

Figura 5. Distribuição da freqüência de ocorrência da queixa "zumbido".

de origem da degeneração da estria ou metabólica da estria vascular. Este tipo de perda geraria mudanças nos potenciais elétricos da cóclea com efeitos possíveis so bre a produção de energia no órgão de Corti, resultando em configuração audiométrica plana, geralmente acompanhada por reconhecimento de fala a bom ${ }^{1}$. Estes dados eliciam a possibilidade de idosos hipertensos apresentarem foco de degeneração na estria vascular, o que justifica apresentarem configuração audiométrica do tipo plana. O utro estudo concorda com este dado revelando que a atrofia da estria vascular pode produzir perdas auditivas de até $60 \mathrm{~dB}$ essencialmente horizontal mesmo em pessoas com todas as células ciliadas preservadas. As causas mais freqüentes das atrofias da estria vascular são doenças metabólicas ${ }^{1}$.

Ainda, dentre os resultados obtidos, pode-se verificar que entre a queixa "Zumbido" e HAS, ocorreu dependência e/ou associação estatística, sendo os não-hipertensos mais propensos a apresentar este tipo de queixa. 0 zumbido geralmente esta associado a perda de células ciliadas da cóclea ${ }^{1} \mathrm{w}$ em alguns estudos com populações idosas foi possível verificar a ocorrência de zumbido em uma parcela relevante da casuística ${ }^{1}$. 0 zumbido é considerado um sintoma e não uma doença e o mecanismo etiológico ainda é incerto, alguns estudos apontam que pode ocorrer em conseqüência de uma atividade neural alterada, resultante de uma lesão ou disfunção em qualquer nível do sistema auditivo ${ }^{1}$. Outros autores sugerem que o zumbido pode ocorrer devido a anormalidades metabólicas, altos níveis de colesterol, traumatismos cranianos e problemas vasculares². 0 zumbido pode ser classificado em objetivo e subjetivo, este último é muito comum em idosos e usualmente descrito como um som ecoante, vibrante ou ressoante ${ }^{21}$.

0 zumbido afeta cerca de $7 \%$ da população e cerca de $1 \%$ consideram-no um problema que compromete significantemente a qualidade de vida ${ }^{2}$. Em estudo com 50 indivíduos hipertensos de 20 a 75 anos o zumbido foi referido 
por $52 \%$ dos casos, fato que não foi observado no presente estudo em que os idosos hipertensos apresentaram apenas $32,4 \%$ de presença de ocorrência deste sintoma. Já em outra pesquisa, em estudo audiológico de 50 indivíduos portadores de hipertensão arterial com idade entre 45 e 63 anos $36 \%$ tiveram como queixas a presença de zumbido ${ }^{22}$, valor mais próximo ao encontrado neste estudo (32,4\%). 0 fato dos idosos hipertensos estudados apresentarem menor quei$x a$ de zumbido pode estar relacionado com o controle da hipertensão, uma vez que to dos os idosos estudados estavam em tratamento com hipertensão controlada, dado que pode ser observado na literatura uma vez que drogas como notrptyne e antidepressivos tricíclicos parecem ter um bom efeito, bem como apoio psicológico sendo o uso de placebos causador de melhora de alguns pacientes com queixa de zumbido ${ }^{1}$

Contudo, neste estudo não observamos relação entre a perda de audição e a hipertensão, uma vez que os achados audiológicos encontrados nos idosos hipertensos e normotensos foram semelhantes. Esse dado concorda com estudos da literatura que buscaram encontrar a ocorrência de perda de audição em pacientes hipertensos controlados e de acordo com os resultados, consideraram que a HAS de larga evolução não provoca deterioração significativa na audição ${ }^{23}$

Finalizando, a hipertensão pode não ser um fator causador da perda de audição, mas, por longos períodos e associada à idade pode agir como um fator somador na deterioração do sistema auditivo.

\section{CONCLUSÃO}

Quando aos achados audiológicos dos grupos estudados pode-se observar que:

- Quanto ao grau da perda de audição os dois grupos apresentaram características semelhantes.

- Com relação à configuração audiométrica o grupo de idosos não-hipertensos apresentou configuração predominantemente do tipo rampa, enquanto que os hipertensos configuração do tipo plana.

- Os idosos não-hipertensos apresentaram maior ocorrência da queixa "zumbido".

\section{REFERÊNCIASBIBLIOGRÁFICAS}

1. Bodanese LC. Hipertensão Arterial no idoso. Rev Bras Clin Ter 1998; 24(5):194-9.
2. American Association of retired persons. A profile of older americans. Washington; 1990.

3. Joint National Committee. The sixth report of the Joint National Committee on prevention detection evaluation and treatment of high blood pressure. Arch Intern Med 1997; (157):2413-46.

4. Sangster JF, Gerace TM, Seewlad RC. Hearing loss in elderly patients in a family practice. Can Med Assoc J 1991; 144(8): 981-4.

5. Russo ICP. Achados audiométricos em uma população de idosos presbiacúsicos brasileiros em função do sexo e da faixa etária. Pró-fono 1993; 5(1): 8-10.

6. Committee on hearing bioacoustics and biomechanics, 1988

7. Bess FH, Williams HA, Lichtentein MJ. Avaliação audiológica dos idosos. Em: Miusiek FE, Rintel-Nann WF. Perspectivas atuais em avaliação audiológica. Manole; 2001. p. 343-69.

8. Lloyd LL, Kaplan H. Audiometric interpretation: a manual of basic audiometry. Baltimore: University Park Press, 1978.

9. Olmos RD, Lotufo PA. Epidemiologia da hipertensão arterial no Brasil e no mundo. Ver Bras Hipertens 2002; 9: 21-3.

10. Miranda RD, Perrotti TC, Bellimazzi VR, Nobrega TM, Cendoroglo MS, Toniolo Neto J. Hipertensão arterial no idoso: peculiaridades na fisiopatologia, no diagnóstico e no tratamento. Ver Bras Hipertens 2002; 9:293-300.

11. Roehe MV, Porsch H, Moussalle S. Presbiacusia. ACTA MED. (POR TO ALEGRE) 1994;15:97-101.

12. Carrasco VN, Prazma J, Faber JE. Cochlear microcirculation effect of adrenergic agonists on arteriole diameter. Arch Otolaryngol Head Neck Surg 1990; (116): 411-7.

13. Leits CMA. A vascular changes in hypertension. Med Clin North Am 1987; 71(5): 827-41.

14. Nazar J, Otarola F, Acevedo L. Audition del paciente hipertenso crônico controlado. Ver Otorrinolaringol Cir Cabeza Cuello 1992; 52: $97-104$

15.Schuknecht HF. Further observations on the pathology of presbycusis. Archives of Otolaryngology 1964; (80): 369 82.

16. Albernaz PLM. Aspectos otoneurológicos da velhice. Acta AWHO 1982; 1(3): 93-5.

17. Bilton T, Ramos, RR, Ebel S, Teixeira LS, Tega LP. Prevalência da deficiência auditiva em uma população idosa. Mundo da Saúde 1997; 21(4): 218-25.

18. Ebel SJ. Prevalência de sintomas e sinais otoneurológicos em pacientes idosos com queixas de tontura [dissertação]. (SP): Universidade Federal de São Paulo; 1994.

19. Ribeiro PJ. Iorio CMM, Fukuda Y. Tipos de zumbido e sua influencia na vida do paciente: estudo em um população ambulatorial. Acta AWHO 2000; 19(3): 125-35.

20. Dossini L, Ravanelli R, Moussalle S. Queixas Otorrinolaringológicas do idoso. Acta Medica [Porto Alegre] 1997; (1): 109-21.

21. Colafemina JF, Grellet M. A função do labirinto anterior e posterior no paciente com hipertensão arterial. Ver Bras De Otorrinolaring 1985; 51(1): 27-30.

22. Brohen VMA, Caovilla HH, Ganança MM. Dos sintomas e achados audiológicos e vestibulares em indivíduos com hipertensão arterial. Acta AWHO 1996; 15(1): 4-10

23. Yan-Lin C, Ding YP. Relationship between hypertension and hearing disorders in the elderly. East African Medical Journal. 1999; 76(6): 344-7. 